\title{
Article
}

\section{Asymmetric Lipid Transfer between Zwitterionic Vesicles by Nanoviscosity Measurements}

\author{
Laure Bar $^{1}$, George Cordoyiannis ${ }^{2}$, Shova Neupane ${ }^{3} \mathbb{D}$, Jonathan Goole ${ }^{4} \mathbb{(}$, Patrick Grosfils ${ }^{5}$ \\ and Patricia Losada-Pérez ${ }^{1, *}$ \\ 1 Experimental Soft Matter and Thermal Physics Group (EST), Department of Physics, \\ Université Libre de Bruxelles, 1050 Brussels, Belgium; laure.bar@ulb.be \\ 2 Condensed Matter Physics Department, Jožef Stefan Institute, 1000 Ljubljana, Slovenia; \\ georgios.kordogiannis@ijs.si \\ 3 Physical Chemistry of Surfaces Group, Institut de Recherche de Chimie Paris (IRCP), 75005 Paris, France; \\ shova.neupane@chimieparistech.psl.eu \\ 4 Laboratory of Pharmaceutics and Biopharmaceutics, Campus de la Plaine, Université Libre de Bruxelles, \\ 1050 Brussels, Belgium; jonathan.goole@ulb.be \\ 5 Center for Nonlinear Phenomena and Complex Systems, Department of Physics, \\ Université Libre de Bruxelles, 1050 Brussels, Belgium; patrick.grosfils@ulb.ac.be \\ * Correspondence: plosadap@ulb.ac.be
}

Citation: Bar, L.; Cordoyiannis, G.; Neupane, S.; Goole, J.; Grosfils, P.;

Losada-Pérez, P. Asymmetric Lipid Transfer between Zwitterionic Vesicles by Nanoviscosity Measurements. Nanomaterials 2021, 11, 1087. https://doi.org/10.3390/ nano11051087

Academic Editor: Ricardo Garcia

Received: 1 April 2021

Accepted: 20 April 2021

Published: 22 April 2021

Publisher's Note: MDPI stays neutral with regard to jurisdictional claims in published maps and institutional affiliations.

Copyright: (c) 2021 by the authors. Licensee MDPI, Basel, Switzerland. This article is an open access article distributed under the terms and conditions of the Creative Commons Attribution (CC BY) license (https:// creativecommons.org/licenses/by/ $4.0 /)$.

\begin{abstract}
The interest in nano-sized lipid vesicles in nano-biotechnology relies on their use as mimics for endosomes, exosomes, and nanocarriers for drug delivery. The interactions between nanoscale size lipid vesicles and cell membranes involve spontaneous interbilayer lipid transfer by several mechanisms, such as monomer transfer or hemifusion. Experimental approaches toward monitoring lipid transfer between nanoscale-sized vesicles typically consist of transfer assays by fluorescence microscopy requiring the use of labels or calorimetric measurements, which in turn require a large amount of sample. Here, the capability of a label-free surface-sensitive method, quartz crystal microbalance with dissipation monitoring (QCM-D), was used to monitor lipid transfer kinetics at minimal concentrations and to elucidate how lipid physicochemical properties influence the nature of the transfer mechanism and dictate its dynamics. By studying time-dependent phase transitions obtained from nanoviscosity measurements, the transfer rates (unidirectional or bidirectional) between two vesicle populations consisting of lipids with the same head group and differing alkyl chain length can be estimated. Lipid transfer is asymmetric and unidirectional from shorter-chain lipid donor vesicles to longer-chain lipid acceptor vesicles. The transfer is dramatically reduced when the vesicle populations are incubated at temperatures below the melting of one of the vesicle populations.
\end{abstract}

Keywords: solid-supported lipid membranes; lipid transfer; quartz crystal microbalance with dissipation monitoring; phase transitions; atomic force microscopy

\section{Introduction}

The interfacial properties of lipid-based assemblies are of major importance for the interbilayer lipid transfer/exchange essential in maintaining the function of cell membranes and in many other biologically relevant processes involving the fusion of membranes in cases of viral infection, hormone, and neurotransmitter release [1-3]. A substantial amount of the current understanding of the molecular mechanisms involving lipid transfer and exchange between membranes has arisen from studies employing model membrane systems due to the complex nature of membranes in vivo. Interbilayer lipid transfer occurs either through vesicular transport in which large amounts of lipids are transferred via protein-mediated fusion of vesicles or through non-vesicular transport by lipid transfer proteins that shuttle lipid monomers between membranes [4,5].

Unlike protein-mediated processes, spontaneous protein-free lipid transfer remains less explored, partly because it is slower and biologically less relevant and requires larger 
activation energies to proceed [6,7]. Nevertheless, spontaneous lipid transfer has been observed in biological processes involving lipid metabolism or parasitic infections $[8,9]$, and its understanding is relevant for the design of lipid-based nanocarriers and for elucidating the active role of lipids in biological processes [10,11]. The kinetics of lipid transfer between two bilayer membranes are intimately related to the thermodynamics of lipid mixing and the lateral organization of their components. These eventually depend on the physicochemical characteristics of the constituent membrane lipids, in particular composition, charge, phase, and the membrane shape geometry (bilayer, vesicle). When two bilayer membranes approach each other, spontaneous lipid transfer can take place by means of several mechanisms: (i) transfer of individual lipids from one membrane into another by diffusion through the aqueous medium, (ii) direct lipid transfer between the apposing membranes without exposure to the aqueous medium, which can happen in cases of short intermembrane separations, and (iii) reorganization of the membrane structure into a hemifused state, where two proximal leaflets partially fuse using a stalk formation, resulting in a rapid lipid exchange between the opposing membranes through lateral diffusion [11-13].

From a purely physicochemical perspective, lipid membranes bearing opposite charges where transfer/exchange proceeds driven by electrostatic interactions, have attracted more attention. The customary methodology for probing spontaneous lipid transfer and exchange is assays involving fluorescently labeled large vesicles at self-quenching concentrations in bulk [14,15]. Apart from bulk methods, surface-sensitive label-free techniques may also be used, such as quartz crystal microbalance with dissipation monitoring (QCM-D) and surface plasmon resonance [16,17]. The former is an acoustic-based technique measuring changes in frequency and energy dissipation of a shear-mode oscillating quartz sensor upon mass adsorption [18]. The shear waves propagate as evanescent waves decaying across the boundary between the sensor and the fluid environment with a penetration depth $\delta=\sqrt{\eta_{L} / \pi f_{n} \rho_{L}}$, depending on the overtone frequency $f_{n}$, on the viscosity $\eta_{L}$ and density $\rho_{L}$ of the fluid in contact with the sensor surface. The penetration depth of a $5 \mathrm{MHz}$ shear wave in water is $\delta \sim 250 \mathrm{~nm}$, rendering QCM-D surface-specific and enabling monitoring changes in the shear viscosity of nanoscale-sized layers. QCM-D has been particularly useful in real-time monitoring of the 'attachment-transfer-detachment' processes for oppositely small charged vesicles onto solid-supported lipid bilayers. In particular, it contributed to elucidating the role of charge in the kinetics of the process $[16,19,20]$. Mechanistically, transfer occurs in both directions between both charged bilayers via monomer transfer or intermediate hemifusion structures [21]. When the lipid molecules have an effective charge, a minimum amount of charged vesicles must adsorb on the supported lipid bilayers (SLB), leading to charge reversal in the latter, indicating that detachment occurs after charge neutralization of the small unilamellar vesicles due to lipid exchange between the two interacting membranes.

Apart from real-time adsorption, the unique sensitivity of QCM-D to mass and energy dissipation changes at the solid-lipid layer-liquid interface enables the detection of phase transformations of solid-supported membrane geometries [22-24]. Upon heating, lipid bilayers change from a more viscous and stiffer gel phase to a less viscous and softer liquiddisordered phase. These changes manifest as anomalies in both frequency and dissipation shift signals and, in particular, in their first-order temperature derivatives $[25,26]$. This feature is advantageous compared to traditional calorimetric methods since it does not require either long temperature equilibration times or a large amount of sample.

Here we assess the capability of QCM-D to detect lipid transfer between nanoscalesized zwitterionic lipid vesicles at a very small concentration, whose interest lies in their use as mimics for endosomes, exosomes, and nanocarriers for drug delivery [27]. Specifically, we focused on detecting lipid transfer taking place between two vesicle populations of zwitterionic saturated phospholipids differing in their alkyl chain lengths-namely, 1,2dimyristoyl-sn-glycero3-phosphocholine (DMPC, chain length $n=14$, melting temperature $T_{m} \sim 24.5^{\circ} \mathrm{C}$ ) and 1,2-dipalmitoyl-sn-glycero-3-phosphocholine (DPPC, $n=16, T_{m} \sim$ $41.5^{\circ} \mathrm{C}$ ). The net transfer kinetics between the vesicles were inferred from their changes in 
phase transition (melting) temperatures with time after the two populations were incubated at a given temperature.

\section{Materials and Methods}

\subsection{Materials Used}

DPPC and DMPC lipids (in powder form) were purchased from Avanti Polar Lipids (Alabaster, AL, USA) and spectroscopic grade chloroform from VWR Chemicals, Leuven, Belgium. The HEPES running buffer was prepared with HEPES powder $\geq 99.5 \%$ (SigmaAldrich, Overijse, Belgium), $\mathrm{NaCl}$ powder $\geq 99 \%$ (Sigma-Aldrich, Overijse, Belgium), and $\mathrm{NaOH}$ powder (VWR chemicals, Leuven Belgium). The masses of lipids were determined gravimetrically using an analytical balance (AG245, Metter-Toledo, Switzerland) with a precision of $\pm 0.1 \mathrm{mg}$. The HEPES buffer solution ( $\mathrm{pH} 7.4$ ) used for the hydration of the dried lipid films was prepared by dissolving $10 \mathrm{mM}$ HEPES and $150 \mathrm{mM} \mathrm{NaCl}$ in Milli-Q water $(18.2 \mathrm{M} \Omega)$. The $\mathrm{pH}$ was then adjusted to 7.4 with $1 \mathrm{M} \mathrm{NaOH}$ solution previously prepared with $\mathrm{NaOH}$ powder and Milli-Q water. To this end, drops of $\mathrm{NaOH}$ solution were added to the buffer during gentle stirring. The $\mathrm{pH}$ value evolution was tracked with a FiveEasy Mettler Toledo pHmeter from Mettler Toledo, Zaventem, Belgium. The buffer was then filtered with $0.2 \mu \mathrm{m}$-pore size PTFE membranes and stored at $4{ }^{\circ} \mathrm{C}$ until being used.

\subsection{Lipid Vesicle Preparation}

Lipids in powder were dissolved in spectroscopic grade chloroform in a roundbottomed flask. The solvent was subsequently evaporated under a continuous mild flow of $\mathrm{N}_{2}$. To avoid any residues of solvent, the lipid films were kept under low pressure overnight. The films were then hydrated with fresh filtered HEPES buffer to $2 \mathrm{mg} / \mathrm{ml}$ under continuous stirring for $45 \mathrm{~min}$ in a temperature-controlled water bath at $60^{\circ} \mathrm{C}$ (well above the melting temperatures of DPPC $T_{m} \sim 41.5^{\circ} \mathrm{C}$ and DMPC $T_{m} \sim 24.5^{\circ} \mathrm{C}$ ). Large unilamellar vesicles (LUVs) were formed by extrusion through filters with different pores of $100 \mathrm{~nm}$ for 25 passes.

The extruded solutions were then diluted in HEPES buffer for obtaining desired concentrations. DMPC and DPPC stock solutions were diluted at $0.1 \mathrm{mg} / \mathrm{ml}$ (to reach a final concentration of $0.05 \mathrm{mg} / \mathrm{ml}$ for each lipid after a 50:50 volume mixing, corresponding to a $0.52 / 0.48$ DMPC/DPPC molar ratio). DMPC and DPPC stock solutions used to inject pure vesicles were directly diluted at $0.05 \mathrm{mg} / \mathrm{mL}$. All solutions were immediately stored in a temperature-controlled incubator (Incu-Line 68R, VWR, Poland; temperature fluctuation $\left.\pm 0.1{ }^{\circ} \mathrm{C}\right)$ at the desired temperatures $\left(T=16^{\circ} \mathrm{C}, T=32{ }^{\circ} \mathrm{C}\right.$, or $\left.\mathrm{T}=50^{\circ} \mathrm{C}\right)$.

\subsection{Dynamic Light Scattering and Zeta Potential Measurements}

The vesicle diameters, polydispersities, and $\zeta$ potentials were determined by means of dynamic light scattering (DLS) (Malvern Zetasizer Nano ZS, Malvern, UK). The obtained mean diameters and polydispersity indexes of the samples used are displayed in Table 1.

Table 1. Hydrodynamic mean diameters and polydispersity indexes (PI) obtained by DLS for the DPPC and DMPC vesicle dispersions used in this work. The number of performed measurements per sample is $n=4$. The calculated errors are the standard deviation of the average values based on several independent solutions.

\begin{tabular}{cccc}
\hline Lipid & Temperature $\left({ }^{\circ} \mathbf{C}\right)$ & Mean Diameter $(\mathbf{n m})$ & PI \\
\hline DPPC & 16 & $119 \pm 6$ & $0.12 \pm 0.06$ \\
DMPC & 16 & $118 \pm 9$ & $0.05 \pm 0.01$ \\
DPPC & 32 & $110 \pm 3$ & $0.08 \pm 0.01$ \\
DMPC & 32 & $120 \pm 3$ & $0.08 \pm 0.01$ \\
DPPC & 50 & $130 \pm 5$ & $0.07 \pm 0.02$ \\
DMPC & 50 & $126 \pm 7$ & $0.09 \pm 0.01$ \\
\hline
\end{tabular}


The mean diameters of DPPC and DMPC vesicles range between 110 and $130 \mathrm{~nm}$, and the maximum recorded polydispersity value is 0.12 , suggesting that vesicle dispersions are homogeneous in size. As previously reported by Enoki et al. [28] for DMPC, the vesicles display a slightly larger mean diameter when lipids are in the liquid disordered phase. For each sample, the zeta-potential was determined in the range between -2.8 and $1.1 \mathrm{mV}$, confirming that DPPC and DMPC vesicles are zwitterionic in the buffer used.

\subsection{Quartz Crystal Microbalance with Dissipation Monitoring}

QCM-D measurements were carried out in a Qsense E4 instrument (Biolin Scientific, Gothenburg, Sweden), which enables monitoring of frequency and dissipation changes, $\Delta f / \mathrm{n}$ and $\Delta D$, with $\mathrm{n}$ the overtone number. This instrument also enables heating or cooling temperature scans in the range between $15^{\circ} \mathrm{C}$ and $65^{\circ} \mathrm{C}$. AT-cut quartz crystals with Au coating (diameter $14 \mathrm{~mm}$, thickness $0.3 \mathrm{~mm}$, quoted surface roughness $<3 \mathrm{~nm}$, and resonant frequency $4.95 \mathrm{MHz}$ ) were used as solid surfaces. The Au-coated quartz sensors were cleaned for $5 \mathrm{~min}$ with a 5:1:1 mixture of Milli-Q water (resistance of $18.2 \mathrm{M} \Omega \mathrm{cm}$ at $25^{\circ} \mathrm{C}$ ), ammonia, and hydrogen peroxide heated at $75^{\circ} \mathrm{C}$, then rinsed in Milli-Q water and dried with $\mathrm{N}_{2}$. Shortly prior to the beginning of the QCM-D measurements, the sensors were exposed to UV-light using a UV-ozone cleaner (Bioforce Nanosciences, Ames, Iowa, USA) for $15 \mathrm{~min}$ and subjected to contact angle measurements before their introduction into the QCM-D modules. The changes in $\Delta f / \mathrm{n}$ and in $\Delta D$ were monitored at five different overtones (from third to eleventh). The running buffer and the lipid vesicles were injected into the QCM-D cells with a flow rate of $50 \mu \mathrm{L} / \mathrm{min}$. The temperature stability was in the order of $\pm 0.02{ }^{\circ} \mathrm{C}$ around the set value. First, a baseline with pure HEPES buffer was established and afterward lipid vesicles were injected over the Au-coated sensor chips. After reaching a stable supported lipid membrane layer (vesicle or bilayer), temperature scans were carried out, upon heating and cooling, at a rate of $0.4^{\circ} \mathrm{C} / \mathrm{min}$, maintaining a 60 min stabilization time between successive temperature ramps.

\subsection{Atomic Force Microscopy (AFM)}

AFM quantitative imaging (QI) and force spectroscopy measurements were performed using a JPK NanoWizard 4 BIO (Bruker, Nano GmbH, Berlin, Germany). All the measurements were done in HEPES buffer at room temperature $\left(T \sim 20^{\circ} \mathrm{C}\right)$. A MLCT-E tip (Bruker, Nano GmbH, Berlin, Germany) with quoted cantilever length of $\sim 0.55 \mu \mathrm{m}$, resonance frequency $\sim 38 \mathrm{kHz}$ and nominal spring constant $\sim 0.1 \mathrm{~N} / \mathrm{m}$ was used. The AFM cantilever was calibrated in buffer against the clean glass slide composing the AFM liquid cell using the equipartition theorem [29]. After QCM-D measurements, samples were immediately transferred to the AFM liquid cell to minimize possible dewetting. QI images were taken on different image sizes using a pixel ratio of either $256 \times 256$ with the tip line rate of $1 \mathrm{~Hz}$, cantilever speeds of 15 and $20 \mu \mathrm{m} / \mathrm{s}$, and setpoint force of $0.2 \mathrm{nN}$. Force mapping was recorded by using force setpoints of 5 and $10 \mathrm{nN}$, with a speed of $1 \mu \mathrm{m} / \mathrm{s}$.

\subsection{Contact Angle Measurements}

Water contact angle (WCA) measurements were performed using an Attension ThetaLite from Biolin Scientific (Gotheburg, Sweden) based on the sessile drop method. A small drop $(3 \mu \mathrm{L})$ of Milli-Q water or diiodomethane was deposited onto clean, UV-ozone treated Au-coated sensors, and the shape of the drop formed on the surface was evaluated. The contact angle of the $3 \mu \mathrm{L}$ droplet of ultrapure water was measured for 10 seconds using a recording speed of $20 \mathrm{frames} / \mathrm{s}$. All contact angles were measured at room temperature. The UV-ozoned Au surfaces were hydrophilic $\left(25^{\circ}<\mathrm{WCA}<34^{\circ}\right)$ as expected.

\section{Results and Discussion QCM-D Results}

DPPC and DMPC vesicle populations were mixed at equivalent mass concentration and incubated for different times (ranging between 0 to $48 \mathrm{~h}$ ) and different temperatures 
$\left(16{ }^{\circ} \mathrm{C}, 32{ }^{\circ} \mathrm{C}\right.$, and $\left.50{ }^{\circ} \mathrm{C}\right)$ before adhesion onto Au-coated quartz surfaces heated at the same three temperatures. The fate of nanoscale lipid vesicles when adsorbing onto a solid sensor is dictated by the interplay between the adhesive energy from lipid-surface interactions and the opposing effect of bending the vesicle bilayer [30,31]. The former depends strongly on the nature of the surface and the latter on the phase of the vesicle constituent lipids. In general, when lipids are injected on Au surfaces, a monotonic frequency decrease (corresponding to mass increase) and a dissipation increase take place for reaching constant non-zero $\Delta f$ and $\Delta D$ plateau values, irrespective of the lipid phase, and indicating the formation of an intact vesicle layer [31]. Figure 1 indicates the plateau values of $\Delta f$ and $\Delta D$ responses (represented for the third overtone) after vesicle adsorption (pure lipids and mixed vesicle populations) on Au-coated quartz sensors at two incubation/adsorption temperatures $\left(T=16\right.$ and $\left.T=50^{\circ} \mathrm{C}\right)$. There was no indication of collective vesicle rupture, and continuous supported lipid bilayer was observed. However, as pointed out by Lind et al. [32], local vesicle rupture events and formation of bilayer patches cannot be ruled out. As a matter of fact, QCM-D is very sensitive to hydrodynamic (wet) mass, and the local, partial formation of SLBs is masked by the adsorption of vesicles on the top or in between the bilayer patches, as was later confirmed by AFM measurements.
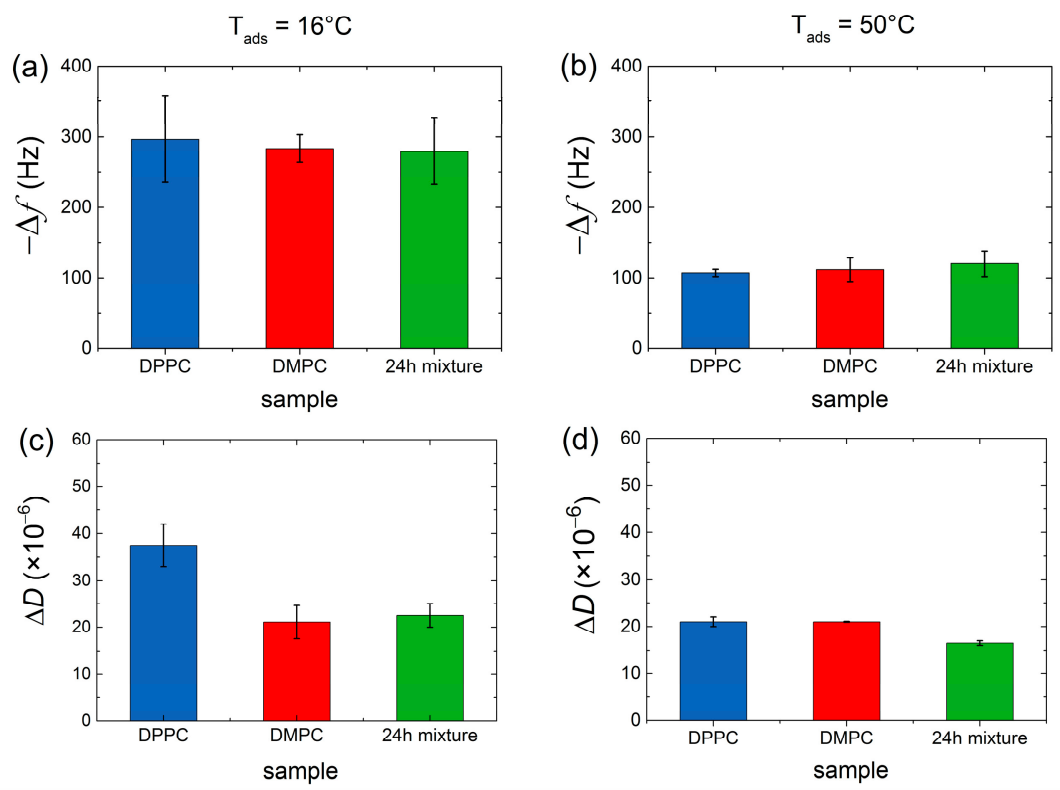

Figure 1. Frequency shifts $\Delta f(\mathbf{a}, \mathbf{b})$ and dissipation shifts $\Delta D(\mathbf{c}, \mathbf{d})$ for the third overtone observed during the adsorption of pure DMPC (red), pure DPPC (blue), and $24 \mathrm{~h}$ incubated DPPC/DMPC mixture (green) at $T_{\text {ads }}=16{ }^{\circ} \mathrm{C}(\mathbf{a}, \mathbf{c})$ and $T_{\mathrm{ads}}=50{ }^{\circ} \mathrm{C}(\mathbf{b}, \mathbf{d})$ on Au-coated quartz sensors. Results obtained at $T_{\text {ads }}=32^{\circ} \mathrm{C}$ can be found in SI (Figure S1).

At $16{ }^{\circ} \mathrm{C}$, the bilayer envelopes of the vesicles are in the gel phase $\left(T<T_{m}\right)$, their bending modulus being $\kappa \sim 10 \times 10^{-19} \mathrm{~J}$, which renders the vesicles quite stiff [33]. At $T=50^{\circ} \mathrm{C}$ the bilayer envelope is in the liquid disordered phase, and the modulus attains a 10 times smaller value of $\kappa \sim 1 \times 10^{-19} \mathrm{~J}$, making vesicles softer and more deformable upon adsorption, thus resulting in a smaller number of vesicles for similar surface coverage. Although vesicles do not rupture globally, attractive interactions to the Au surface induce deformation, and vesicles deform leading to a smaller thickness than in bulk, and even rupture locally, forming bilayer patches. At $T=32{ }^{\circ} \mathrm{C}$, bilayer envelopes of DMPC vesicles are in their liquid phase, while the occurrence of periodic ripples in DPPC lipid bilayers is very likely. The frequency shifts plateau value $\Delta f_{3} \sim-179 \mathrm{~Hz}$ resulting from pure DMPC vesicles adsorption is in between those observed at $T=16{ }^{\circ} \mathrm{C}$ and $T=50^{\circ} \mathrm{C}$ (see Figure S1). Pure DPPC and the mixed vesicle population display frequency shift values closer to the ones observed at $T=16{ }^{\circ} \mathrm{C}$ since DPPC lipids at $T=32{ }^{\circ} \mathrm{C}$ are mostly in the gel phase. 
After the vesicle/bilayer layer formation on the Au-coated sensors, subsequent heating and cooling cycles were carried out at a rate of $0.4^{\circ} \mathrm{C} / \mathrm{min}$ to assess the shifts in melting temperature resulting from lipid transfer between the two vesicle populations. Figure 2 displays the first-order temperature derivatives of $\Delta f(T)$ signals upon heating for vesicle populations of pure DPPC, pure DMPC, as well as DMPC and DPPC incubated for $24 \mathrm{~h}$ at different temperatures. The main phase transitions are identified by extrema (maxima) in the $d \Delta f(T) / d T$ responses. The main transitions for pure lipid DPPC or DMPC vesicles are characterized by large and rather sharp peaks located at temperatures in agreement with those reported in the literature using similar heating rates [31,34]. The main transitions were also observed upon cooling with a hysteresis of $\Delta T_{m} \sim 2$ to $3{ }^{\circ} \mathrm{C}$, characteristic of first-order phase transitions [35] (an example of the hysteresis effect is shown in Figure S2 of the Supplementary Material). The phase transition of pure DMPC when incubated at low temperatures displays a double-peak behavior. The presence of the two peaks has been previously observed in the literature from calorimetric and QCM-D measurements, and it is a direct consequence of the extrusion process and related to structural transitions in the vesicle itself $[36,37]$.

(a)

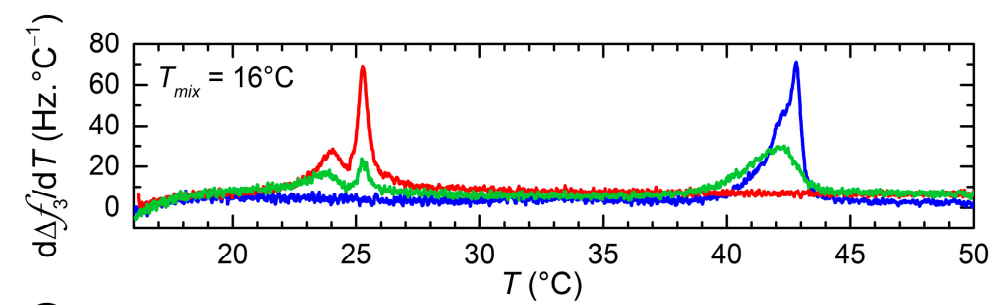

(b)

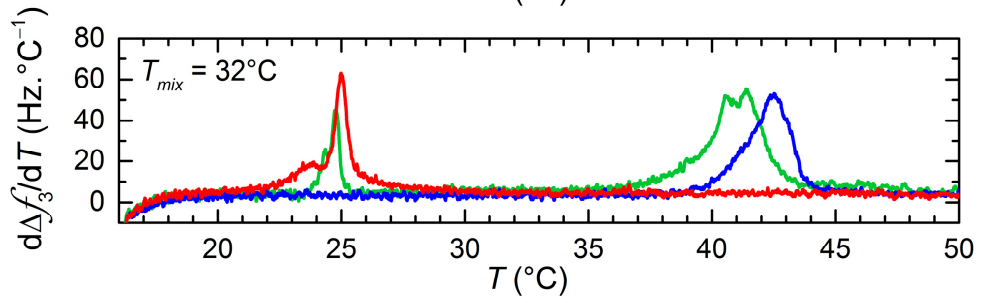

(c)

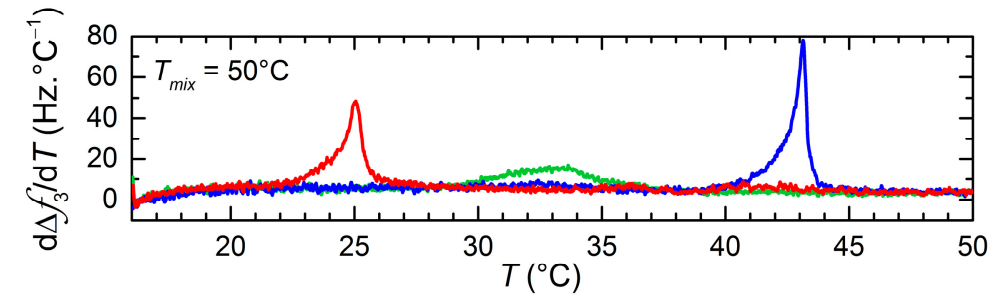

Figure 2. Temperature dependence of $\mathrm{d} \Delta f_{3} / \mathrm{d} T$ (third overtone) upon the first heating for pure DMPC LUVs (red), pure DPPC LUVs (blue), and $24 \mathrm{~h}$ DPPC/DMPC mixture (green) adsorbed at $T=16{ }^{\circ} \mathrm{C}(\mathbf{a}), T=32{ }^{\circ} \mathrm{C}(\mathbf{b})$, and $T=50{ }^{\circ} \mathrm{C}(\mathbf{c})$ on Au-coated quartz surfaces.

As regards the mixtures of DMPC and DPPC vesicle populations incubated for $24 \mathrm{~h}$, a significantly different behavior is observed depending on the incubation temperature. At $T=16^{\circ} \mathrm{C}$, both DMPC and DPPC lipids are in the gel phase, and the location of the main transition peaks is practically unaffected. At $T=32{ }^{\circ} \mathrm{C}, \mathrm{DMPC}$ is in the liquid-disordered phase, while DPPC is beginning the 'gel to ripple' phase transition, the majority of lipid molecules being in the gel phase. The location of the low-T peak remains unchanged, whereas the high-T peak is shifted toward lower temperatures, indicating DMPC lipid transfer into DMPC vesicles. At $T=50^{\circ} \mathrm{C}$, both lipids are in the liquid-disordered phase. After $24 \mathrm{~h}$ incubation, the low-T peak is not appearing anymore, whereas the high-T peak is significantly broadened and shifted toward lower temperatures.

Figure 3 provides a three-dimensional overview of the phase transition peak evolution as a function of incubation time and temperature, with the aim to get more insights into the nature (symmetric or asymmetric) of lipid transfer between the vesicles adsorbed onto 
the Au surfaces. At the beginning of the incubation period $(t \sim 0.5 \mathrm{~h})$, two clear peaks can be distinguished for vesicle populations incubated at all three temperatures. At $T=16{ }^{\circ} \mathrm{C}$ (panel a) and $\mathrm{T}=32{ }^{\circ} \mathrm{C}$ (panel b), the location of the transition temperatures of the mixed vesicle populations remains close to their pure lipid counterparts, while the peak intensities are reduced. The reduction in peak intensity can be attributed to (i) limited lipid transfer taking place and transitions becoming broader and (ii) local vesicle rupture occurring upon adsorption (the peak intensity scales with the mass adsorbed, including coupled water trapped within intact vesicles). At $T=50{ }^{\circ} \mathrm{C}$ (panel c), the low temperature peak is located very close to the phase transition of pure DMPC, while the high temperature peak is significantly shifted as compared with pure DPPC melting temperature $\left(\Delta T_{m} \sim-4.4^{\circ} \mathrm{C}\right)$. With increasing incubation time, the original transition of the DPPC vesicle population is shifted to lower temperatures, whereas the low temperature peak remains at the same location and vanishes with time (see Figure S3 in the Supplementary Materials). The DMPC vesicle population is continuously depleted of its lipids until the vesicles become unstable and equimolarity is reached. This process was completed from $24 \mathrm{~h}$ incubation after which the no significant shifts in the transition peak were observed. As a matter of fact, the temperature interval where the transition takes place shows a good agreement with previously reported transitions of equimolar mixtures of DMPC and DPPC vesicles formed with lipids previously mixed [38].

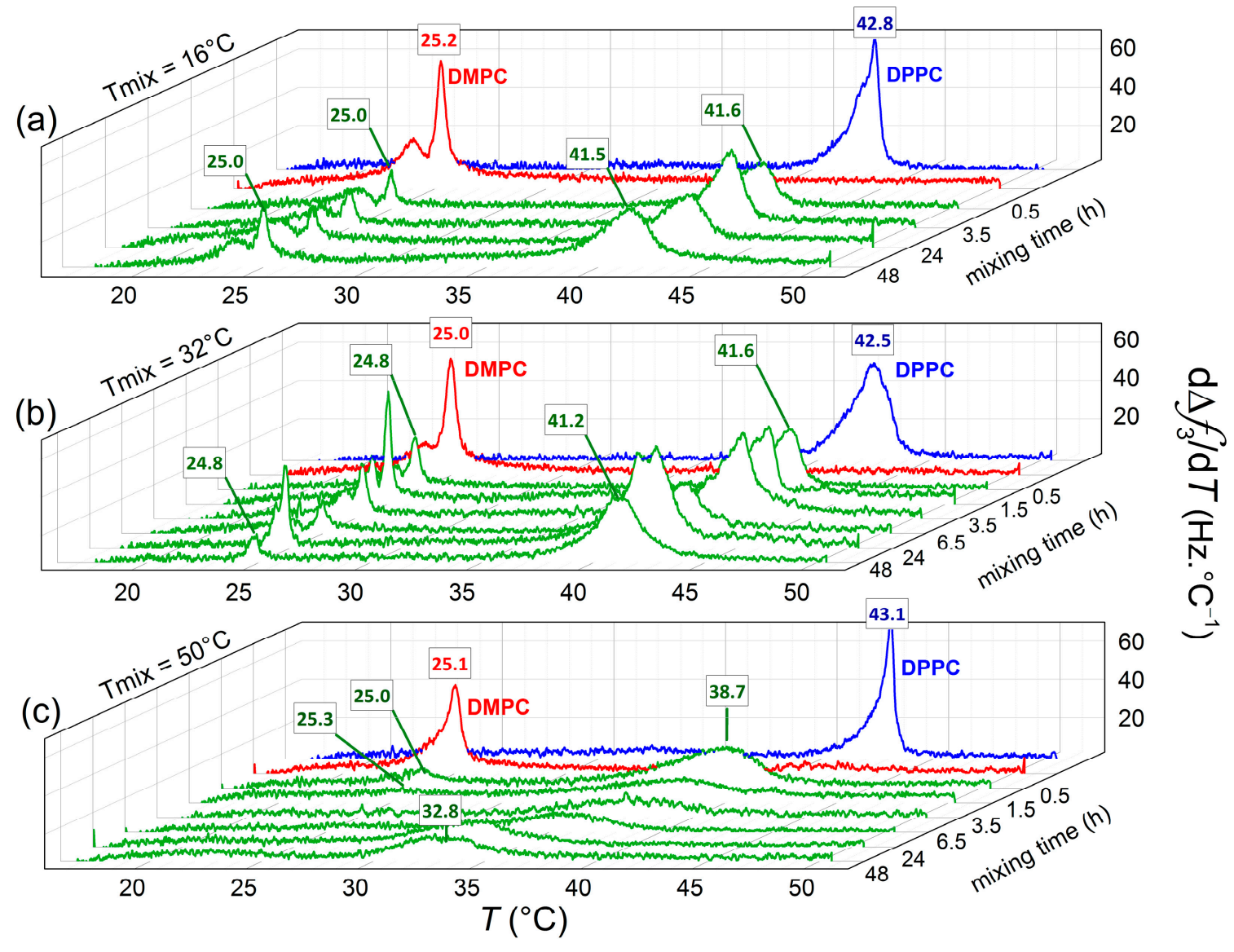

Figure 3. Dependence of the mixing time on the main phase transition parameters of adsorbed vesicles on Au-coated quartz surfaces. $\mathrm{d} \Delta f / \mathrm{d} T$ curves obtained upon heating demonstrate the phase transitions of samples at $T=16{ }^{\circ} \mathrm{C}(\mathbf{a}), T=32{ }^{\circ} \mathrm{C}(\mathbf{b})$, and $T=50^{\circ} \mathrm{C}(\mathbf{c})$.

For the sake of clarity regarding the melting temperature shifts observed in Figure 3, we plotted the mixing time dependence of the main phase transitions at the three incubation temperatures for the high- $T$ (Figure 4a) and low- $T$ peaks (Figure $4 \mathrm{~b}$ ). At $T=16^{\circ} \mathrm{C}$ and 
$T=32{ }^{\circ} \mathrm{C}$, the displayed time-dependent $T_{m}$ values deviate only slightly from the ones of pure lipids. Conversely, at $T=50^{\circ} \mathrm{C}$, a continuous decrease is observed for the high-T peak, while the low-T peak displays a very mild increase, indicating that asymmetric lipid transfer takes place from DMPC vesicles (donor) toward DPPC vesicles (acceptor).
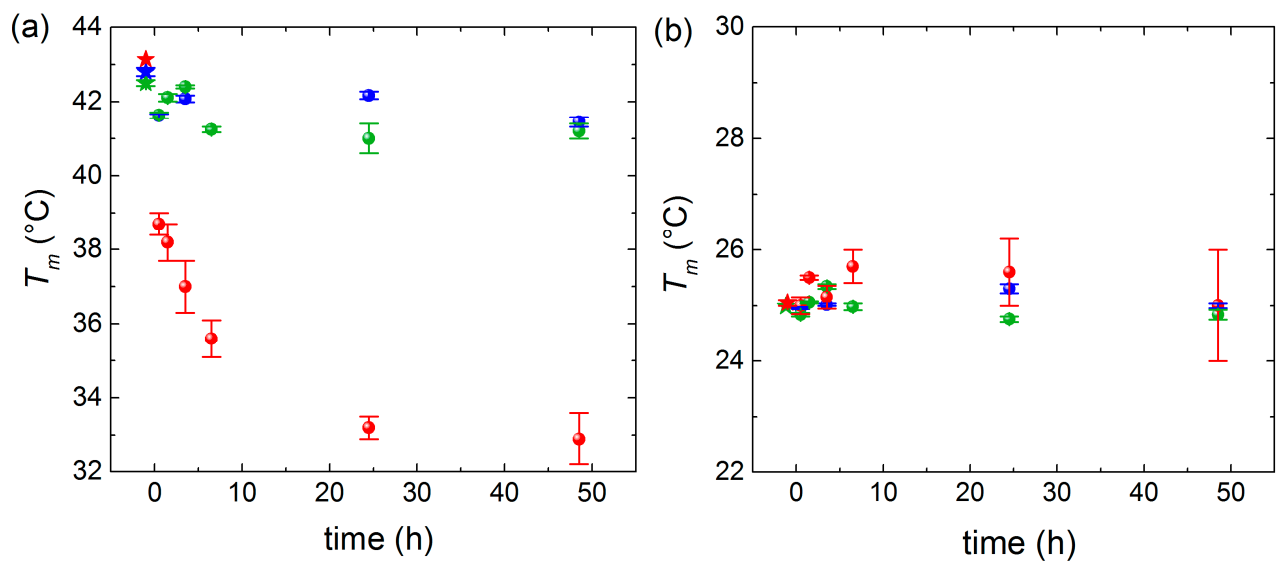

Figure 4. Dependence of the incubation time on $T_{m}$ values extracted from (a) high- $T$ peaks and (b) low-T peaks (blue points for $T=16^{\circ} \mathrm{C}$, green points for $32{ }^{\circ} \mathrm{C}$, red points for $50{ }^{\circ} \mathrm{C}$ ). Stars represent $T_{m}$ of pure DPPC vesicle (a) and pure DMPC vesicles (b), which are used as reference.

In order to get insights into the kinetics of lipid transfer, we analyzed our results in the framework of a kinetic model introduced by Thilo [39] and later applied by Bayerl et al. [40] on DMPC-DPPC exchange of small vesicles using calorimetric measurements. Bayerl et al. reported that in the case of small sonicated vesicles (diameter $\sim 60 \mathrm{~nm}$ ), lipid transfer took place by monomer transfer from DMPC to DPPC vesicles, whereas larger vesicles (200 $\mathrm{nm}<$ diameter $<900 \mathrm{~nm}$ ) prepared by detergent dialysis exchanged lipids mainly by vesicle fusion. The conclusions were based on the shape and evolution of transition peaks from heat capacity curves of vesicle populations incubated at $T=45^{\circ} \mathrm{C}$. The former displayed $T_{m}$ shifts of the high-T peak, while the latter both high- $T$ and low- $T$ remained non-shifted with time, with a broader peak located in between the unshifted peaks arising and coexisting at longer incubation times. Our results coincide with the results previously reported for small sonicated vesicles; thus, we conclude that lipid transfer in our systems takes place rather by monomer transfer from DMPC to DPPC vesicles. During spontaneous lipid transport, DMPC first desorbs from the donor vesicle, diffuses through the solvent (buffer), and enters the DPPC acceptor membrane. The model of Thilo assumes that (a) the rate-limiting step is the monomer desorption from the donor vesicles, and (b) the rate at which lipid monomers in the bulk solution are taken by the acceptor vesicles is proportional to the product of the monomer concentration and the total bilayer surface area in a unit volume. It considers two vesicle populations I and II and assumes that at time zero $(t=0)$ the former contains only DPPC and the latter DMPC. After an incubation time $t$, the transition temperatures $T^{I}(t)$ and $T^{I I}(t)$ of vesicle populations I and II can be expressed as:

$$
\begin{aligned}
T^{I}(t) & =x^{I}\left(T_{m}^{D M P C}-T_{m}^{D P P C}\right)+T_{m}^{D P P C} \\
T^{I I}(t) & =x^{I I}\left(T_{m}^{D M P C}-T_{m}^{D P P C}\right)+T_{m}^{D P P C}
\end{aligned}
$$

where $x^{I}$ and $x^{I I}$ are the molar fractions of DMPC in vesicle populations I and II, respectively. From the values of $T^{I}(t)$ and $T^{I I}(t)$, the off rate constants of DMPC and DPPC dissociated from their original vesicle populations can be obtained:

$$
x^{I}(t)=\frac{1-e^{-y t}}{\frac{1}{r}-\left(1-k_{D P P C}^{o f f} / k_{D M P C}^{o f f}\right) e^{-y t}}
$$




$$
x^{I I}(t)=\frac{(1-r) k_{D M P C}^{o f f} e^{-y t}+r k_{D P P C}^{o f f}}{(1-r)\left(k_{D M P C}^{o f f}-k_{D P P C}^{o f f}\right) e^{-y t}+k_{D P P C}^{o f f}}
$$

with $y=r k_{D P P C}^{o f f}+(1-r) k_{D M P C}^{o f f}$ and $r=[D M P C] /[(D M P C]+[D P P C])$. In our particular case, $r=0.52$ and $k_{D P P C}^{o f f}=0$, assuming asymmetric unidirectional lipid transfer. Our assumption is based on the fact that the lipid desorption from the donor vesicles is the ratelimiting step and determines the activation energy of lipid transfer. As recently reported by Rogers et al. [41], the membrane hydrophobicity dictates the activation energy for lipid transfer. The energy cost for desorption from the donor vesicle increases as the lipid acyl chain increases and membrane order increases. Experimentally, activation energies for interbilayer monomer transfer and transbilayer (flip-flop) transfer can be accurately determined by time-resolved small angle neutron scattering, as used by Nakano et al. for symmetric transfer between DMPC LUVs [42]. In our particular case, DPPC being longer than DMPC and in the gel phase, its activation energy is larger and thus less likely for monomer desorption. Figure 5 displays the calculated mole fraction of DMPC into DPPC vesicles incubated at $50{ }^{\circ} \mathrm{C}$ as a function of time, together with its corresponding fit to Equation (3). The resulting off rate constant $k_{D M P C}^{o f f}=(16 \pm 4) \times 10^{-5} \mathrm{~s}^{-1}$ and half-time for $50 \%$ lipid transfer $t_{1 / 2}=1.46 \pm 0.04 \mathrm{~h}$ agree very well with reported calorimetric results by Bayerl et al. As expected, the off rates obtained for samples incubated at $16{ }^{\circ} \mathrm{C}$ and $32{ }^{\circ} \mathrm{C}$ are considerably reduced, and accordingly, the half-times are dramatically increased. At $T=16^{\circ} \mathrm{C}$, both DMPC and DPPC lipids are in the gel phase, and transfer is limited by the decreased desorption rate of DMPC monomers from the donor vesicles. At $T=32{ }^{\circ} \mathrm{C}$, DMPC is in the liquid disordered phase, and thus a faster DMPC monomer desorption from the donor vesicles occurs. However, the transfer is very likely limited by the reduced monomer insertion rate due to the fact that DPPC is mostly in the gel phase. For the sake of clarity, a summary of the (asymmetric) transfer detected by QCM-D is schematically depicted in Figure 6. The results at low temperatures agree qualitatively with calorimetric results in that transfer is significantly reduced when lipids are in the gel phase. A quantitative comparison is out of the scope of this paper since additional data at higher incubation times $(t>48 \mathrm{~h})$ would be necessary in order to achieve precise fits for mixing at $32{ }^{\circ} \mathrm{C}$ and $16{ }^{\circ} \mathrm{C}$.

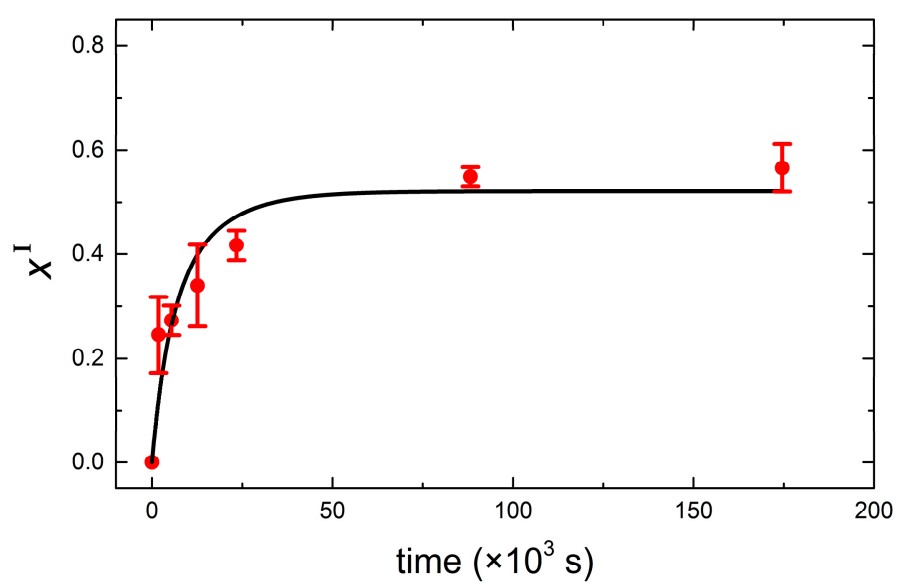

Figure 5. Mole fraction of DMPC in vesicle population I (DPPC vesicles) as a function of time. Red solid dots are experimental points, and the black solid line is the fit to Equation (3).

After the first thermal (heating and cooling) cycle, subsequent cycles were carried out to assess the effect of crossing the phase transition on the net transfer. Figure 7 shows the dependence of phase transition behavior upon thermal cycling for vesicle dispersions of DMPC and DPPC incubated at $16^{\circ} \mathrm{C}$ during $24 \mathrm{~h}$. During the first heating, two peaks corresponding to the melting of two independent vesicle populations could be clearly 
observed, indicating the absence of lipid transfer at low temperatures. During the second heating a broad single peak appears, which becomes sharper with subsequent thermal cycles, indicating that crossing the main transition accelerates the lipid transfer between the two vesicle populations. A similar pattern of behavior can be seen for vesicles incubated at $32{ }^{\circ} \mathrm{C}$ (see Figure $\mathrm{S} 4$ in the Supplementary Materials). Since during the thermal cycles, vesicles are adsorbed on the Au surface, we are inclined to think that transfer occurs in this case by vesicle fusion, induced by the change in mechanical properties of adsorbed vesicles (changing from a stiffer to a softer state). Vesicle populations incubated at $50{ }^{\circ} \mathrm{C}$ display no significant changes upon thermal cycling since transfer until equimolarity had already occurred in bulk (see Figure S4 in the Supplementary Materials).

Step 1: Bulk incubation
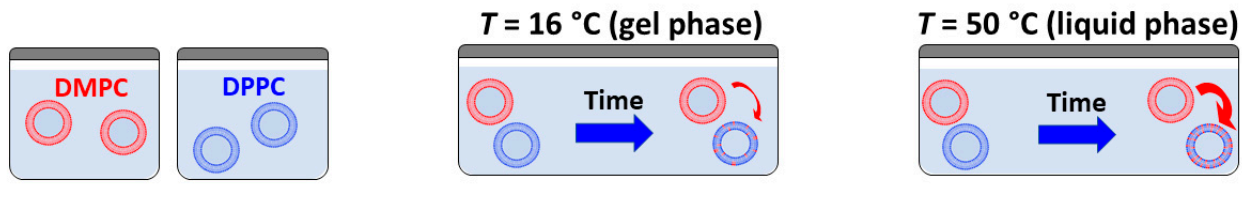

Step 2: QCM-D measurements
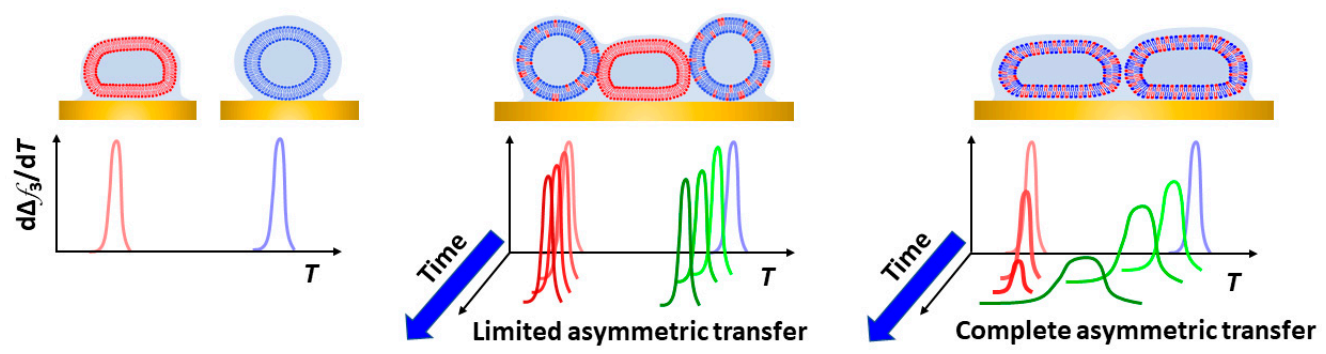

Figure 6. Schematic representation of lipid transfer detected by QCM-D nanoviscosity measurements.

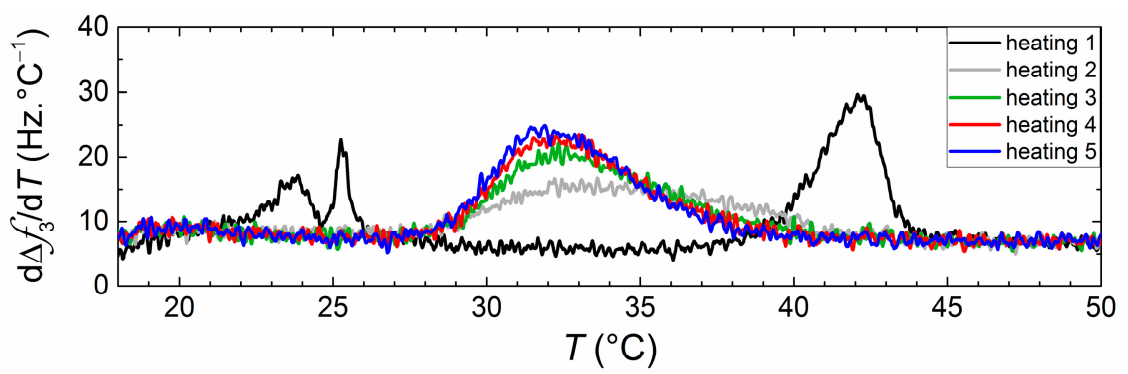

Figure 7. $\mathrm{d} \Delta f_{3} / \mathrm{d} T$ curves obtained upon successive heatings of the DPPC and DPPC vesicle dispersions incubated for $24 \mathrm{~h}$ at $\mathrm{T}=16^{\circ} \mathrm{C}$.

Immediately after the thermal cycles were carried out in the QCM-D flow cells, the Aucoated QCM-D sensors with the supported lipid layers were transferred to an AFM liquid cell. The QCM-D sensors were kept submerged in buffer at all times to ensure that the lipid layers were always hydrated. The surface coverage of the Au-sensors with the lipid layers was assessed by QI-mode imaging at low force setpoint, followed by force mapping at higher force setpoints. A freshly cleaned gold-coated QCM-D sensor was used as reference. Figure S5 of the Supplementary Materials displays the topography of a bare Au sensor (a) as well as pure DMPC, pure DPPC, and adsorbed vesicles incubated at $50^{\circ} \mathrm{C}$ for $24 \mathrm{~h}$ in HEPES buffer (b). The bare Au surface is rather flat (RMS roughness $=0.95 \pm 0.05$ ) with polycrystalline texture with grains of lateral size $\sim 80 \mathrm{~nm}$ as recently shown [43]. Surfaces with adsorbed lipid layers appear more inhomogeneous, displaying both supported lipid bilayers and intact adsorbed vesicle layers. As reported by Lipowsky and coworkers [44], 
homogeneous coverage of lipid layers is limited on polycrystalline surfaces, while it can take place on large Au grains with atomically flat (111) terraces. Unlike layers formed onto atomically flat surfaces such as mica, the underlying substrate roughness prevents distinguishing whether the formed layers are bilayers, monolayers, or multilayers by solely inspecting the height measured of the layers. The presence of each type of layer was evaluated by the shape of force curves recorded at different spots of each sample. Samples containing pure DMPC consisted of $64 \%$ bilayers, $26 \%$ intact vesicles, and 10\% multilayers. Intact vesicles could have fused and ruptured during the thermal cycling by thermal stress. Pure DPPC consisted of $12 \%$ bilayers, $85 \%$ intact vesicles, and $4 \%$ multilayers. Vesicle populations of DMPC and DPPC incubated $24 \mathrm{~h}$ at $50{ }^{\circ} \mathrm{C}$ displayed a composition of $45 \%$ bilayers, $50 \%$ intact vesicles, and 5\% multilayers. For simplicity, we will only evaluate the nanomechanical properties of supported lipid bilayers.

Figure 8 displays representative force curves as a function of the tip-sample separation distance $d$ during approach. The zero-separation distance $d=0$ is defined as the point where the tip comes into hard contact with the surface. Upon approaching the AFM tip to the lipid-covered Au surface, no interaction is observed until $d \sim 15 \mathrm{~nm}$ tip-surface vertical separation distance, when repulsive interactions between the tip and the lipid covered surface take over. From that point, the supported lipid layer is elastically compressed until the tip punches through the layer and enters into contact with the Au surface. The discontinuity in the approaching force distance curve stands as a token of the penetration of the AFM tip through the lipid bilayer. The vertical force at which this discontinuity takes place corresponds to the maximum force that the bilayer is able to withstand before breaking, commonly referred to as the breakthrough force $\left(F_{\mathrm{b}}\right)[45,46]$. The tip-sample distance at which penetration occurs relates to the thickness of the lipid layer formed. In all cases, the average tip-sample distance falls within 4 to $5 \mathrm{~nm}$, which agrees well with the typical thickness of compressed supported lipid bilayers. Interestingly, the average value breakthrough force $F_{\mathrm{b}}$ shows a clear trend, the system where lipid transfer has taken place displaying an intermediate value between pure DMPC and pure DPPC (see inset Figure 7). The corresponding statistical analysis for the jump thickness can be found in Figure S6 of the Supplementary Material. It is worth mentioning that all samples were measured at room temperature $\left(T \sim 20^{\circ} \mathrm{C}\right)$, where both DMPC and DPPC lipid bilayers are in the gel phase. The larger value of $F_{\mathrm{b}}$ for DPPC can be ascribed to the increased hydrophobic interactions due to longer alkyl chains.

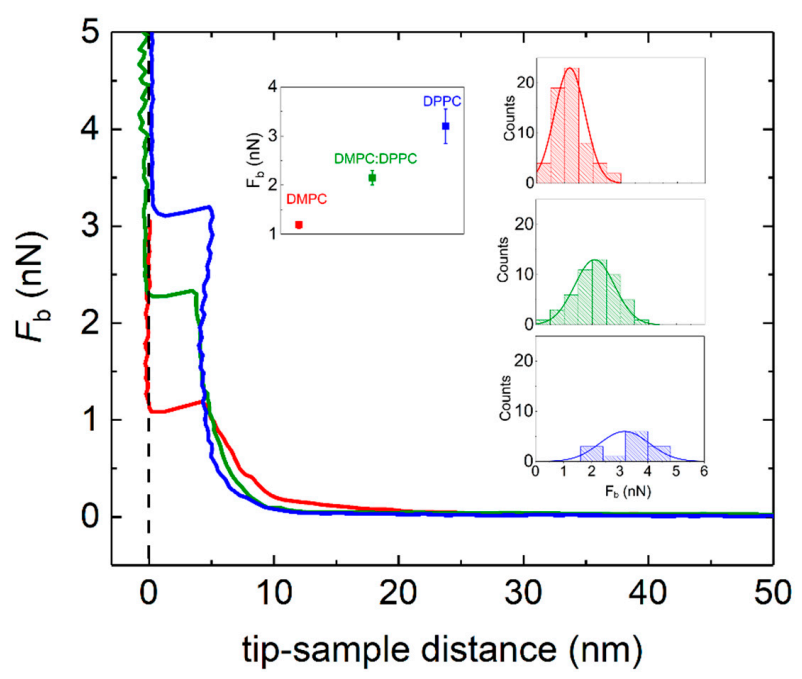

Figure 8. Approach force-distance curves on DMPC (red solid line), DMPC:DPPC (green solid line), and DPPC (blue solid line) supported lipid bilayers on Au-coated QCM-D sensors. Inset: Corresponding histograms taken from a given number of force-distance curves. 


\section{Conclusions}

In this work we have demonstrated the capability of QCM-D to monitor the dynamics of lipid transfer between nanoscale-sized lipid vesicles consisting of zwitterionic lipids differing only in two ethyl groups-namely, DMPC and DPPC. Lipid transfer kinetics was assessed by time-dependent changes in viscoelastic properties of the supported membrane layers during their main phase transition. Lipid transfer proceeds in an asymmetric manner, from donor (DMPC) to acceptor (DPPC) vesicles by lipid monomer transfer. Our measurements reflect the influence of lipid physicochemical and related mechanical properties on the kinetics of lipid transfer. When both types of vesicles are in the gel phase, transfer is very slow and limited by the reduced desorption and insertion rates. When both types of vesicles are in the liquid disordered phase, transfer proceeds faster and kinetic rates agree well with those obtained by calorimetry. Thermal cycling through the transition accelerates the transfer, even for vesicle populations that were originally incubated at gel phases. Complementary AFM measurements provide nanomechanical signatures of supported lipid bilayers where transfer has taken place. Homogeneously mixed layers display breakthrough forces in between those observed for their pure short-chain and long-chain counterparts.

Overall, lipid transfer kinetics assessed by changes in viscoelastic properties of the adsorbed lipid layers are in agreement with calorimetry measurements and demonstrate the potential nano of QCM-D for detecting and quantifying lipid transfer between zwitterionic nanoscale-sized vesicles with high sensitivity and reduced amounts of biomolecules. We anticipate additional measurements of transfer in situ to shed more light on the role of layer topography in transfer kinetics.

Supplementary Materials: The following are available online at https:/ /www.mdpi.com/article/10 $.3390 /$ nano11051087/s1. Figure S1: Frequency shifts (a) and dissipation shifts (b) observed during the adsorption of pure DMPC (red), pure DPPC (blue), and $24 \mathrm{~h}$ DPPC/DMPC mixture (green) at $32{ }^{\circ} \mathrm{C}$ on Au quartz sensors; Figure S2: Temperature dependence of $\mathrm{d} \Delta f_{3} / \mathrm{d} T$ (3rd overtone) upon cooling and heating for pure DMPC LUVs and pure DPPC LUVs adsorbed at $50{ }^{\circ} \mathrm{C}$ on Au-coated quartz surfaces; Figure S3: Dependence of the mixing time ( $30 \mathrm{~min}, 1 \mathrm{~h} 30 \mathrm{~min}, 3 \mathrm{~h} 30 \mathrm{~min}, 6 \mathrm{~h}$ $30 \mathrm{~min}, 24 \mathrm{~h}$, or $48 \mathrm{~h}$ ) on the main phase transition temperature of adsorbed vesicles on Au-coated quartz surfaces. $\mathrm{d} \Delta f / \mathrm{d} T$ curves obtained upon heating demonstrate the phase transitions of samples incubated at $50{ }^{\circ} \mathrm{C}$ (c). Figure S4: $\mathrm{d} \Delta f / \mathrm{d} T$ curves obtained from successive heatings of the DPPC and DMPC vesicle dispersions incubated for $24 \mathrm{~h}$ at $T_{\text {inc }}=16{ }^{\circ} \mathrm{C}, 32{ }^{\circ} \mathrm{C}$, and $50{ }^{\circ} \mathrm{C}$; Figure S5: Height measured of a bare Au sensor (a) as well as pure DMPC, pure DPPC, and adsorbed vesicles incubated at $50{ }^{\circ} \mathrm{C}$ for $24 \mathrm{~h}$ in HEPES buffer (b); Figure S6: Statistical analysis of the jump thickness upon lipid bilayer perforation. The histograms correspond to a given number of force curves taken on DMPC (red solid line), DMPC:DPPC (green solid line), and DPPC (blue solid line) supported lipid bilayers on Au-coated QCM-D sensors.

Author Contributions: Conceptualization, L.B., G.C., P.G., and P.L.-P.; data acquisition, L.B., J.G., and P.L.-P.; QCM-D data analysis, L.B. and G.C.; AFM data analysis, L.B. and S.N.; writing-original draft preparation, L.B., G.C., and P.L.-P.; writing-review and editing, all authors; supervision, G.C., P.G., and P.L.-P.; funding acquisition, P.L.-P. All authors have read and agreed to the published version of the manuscript.

Funding: This research was funded by the Université Libre de Bruxelles (ULB), Project ARC grant number 20061, and the Fonds de la Recherche Scientifique (FNRS) Project MIS under grant number F.4525.20. G.C. acknowledges the financial support of the Slovenian Research Agency, Project P1-0125.

Data Availability Statement: The data presented in this study are available from the corresponding author upon request.

Conflicts of Interest: The authors declare no conflict of interest. 


\section{References}

1. Toulmay, A.; Prinz, W.A. Lipid transfer and signaling at organelle contact sites: The tip of the iceberg. Curr. Opin. Cell Biol. 2011, 23, 458-463. [CrossRef]

2. Mazzon, M.; Mercer, J. Lipid interactions during virus entry and infection. Cell. Microbiol. 2014, 16, 1493-1502. [CrossRef] [PubMed]

3. Postila, P.A.; Róg, T. A Perspective: Active Role of Lipids in Neurotransmitter Dynamics. Mol. Neurobiol. 2020, 57, 910-925. [CrossRef]

4. Stefan, C.J.; Trimble, W.S.; Grinstein, S.; Drin, G.; Reinisch, K.; De Camilli, P.; Cohen, S.; Valm, A.M.; Lippincott-Schwartz, J.; Levine, T.P.; et al. Membrane dynamics and organelle biogenesis-lipid pipelines and vesicular carriers. BMC Biol. 2017, 15, 102. [CrossRef]

5. Reinisch, K.M.; Prinz, W.A. Mechanisms of nonvesicular lipid transport. J. Cell Biol. 2021, 220, 202012058. [CrossRef] [PubMed]

6. Brown, R.F. Spontaneous lipid transfer between organized lipid assemblies. Biochim. et Biophys. Acta (BBA) - Rev. Biomembr. 1992, 1113, 375-389. [CrossRef]

7. Richens, J.L.; Tyler, A.I.I.; Barriga, H.M.G.; Bramble, J.P.; Law, R.V.; Brooks, N.J.; Seddon, J.M.; Ces, O.; O'Shea, P. Spontaneous charged lipid transfer between lipid vesicles. Sci. Rep. 2017, 7, 12606. [CrossRef] [PubMed]

8. Golan, D.E.; Furlong, S.T.; Brown, C.S.; Caulfield, J.P. Monopalmitoylphosphatidylcholine incorporation into human erythrocyte ghost membranes causes protein and lipid immobilization and cholesterol depletion. Biochemistry 1988, 27, 2661-2667. [CrossRef] [PubMed]

9. $\quad$ Staggers, J.E.; Hernell, O.; Stafford, R.J.; Carey, M.C. Physical-chemical behavior of dietary and biliary lipids during intestinal digestion and absorption. 1. Phase behavior and aggregation states of model lipid systems patterned after aqueous duodenal contents of healthy adult human beings. Biochemistry 1990, 29, 2028-2040. [CrossRef]

10. Somerharju, P. Is Spontaneous Translocation of Polar Lipids Between Cellular Organelles Negligible? Lipid Insights 2015, 8s1, 87-93. [CrossRef] [PubMed]

11. Nichols, J.W.; Pagano, R.E. Kinetics of soluble lipid monomer diffusion between vesicles. Biochemistry 1981, 20, 2783-2789. [CrossRef]

12. Zhu, T.; Jiang, Z.; Ma, Y. Lipid exchange between membranes: Effects of membrane surface charge, composition, and curvature. Colloids Surfaces B: Biointerfaces 2012, 97, 155-161. [CrossRef] [PubMed]

13. Lei, G.; Macdonald, R.C. Lipid Bilayer Vesicle Fusion: Intermediates Captured by High-Speed Microfluorescence Spectroscopy. Biophys. J. 2003, 85, 1585-1599. [CrossRef]

14. Elvington, S.M.; Nichols, J.W. Spontaneous, intervesicular transfer rates of fluorescent, acyl chain-labeled phosphatidylcholine analogs. Biochim. et Biophys. Acta (BBA) - Biomembr. 2007, 1768, 502-508. [CrossRef] [PubMed]

15. Lira, R.B.; Robinson, T.; Dimova, R.; Riske, K.A. Highly Efficient Protein-free Membrane Fusion: A Giant Vesicle Study. Biophys. J. 2019, 116, 79-91. [CrossRef] [PubMed]

16. Kunze, A.; Sjövall, P.; Kasemo, B.; Svedhem, S. In Situ Preparation and Modification of Supported Lipid Layers by Lipid Transfer from Vesicles Studied by QCM-D and TOF-SIMS. J. Am. Chem. Soc. 2009, 131, 2450-2451. [CrossRef]

17. Margheri, G.; D’Agostino, R.; Becucci, L.; Guidelli, R.; Tiribilli, B.; Del Rosso, M. Surface plasmon resonance as detection tool for lipids lateral mobility in biomimetic membranes. Biomed. Opt. Express 2012, 3, 3119-3126. [CrossRef] [PubMed]

18. Reviakine, I.; Johannsmann, D.; Richter, R.P. Hearing What You Cannot See and Visualizing What You Hear: Interpreting Quartz Crystal Microbalance Data from Solvated Interfaces. Anal. Chem. 2011, 83, 8838-8848. [CrossRef] [PubMed]

19. Wikström, A.; Svedhem, S.; Sivignon, M.; Kasemo, B. Real-Time QCM-D Monitoring of Electrostatically Driven Lipid Transfer between Two Lipid Bilayer Membranes. J. Phys. Chem. B 2008, 112, 14069-14074. [CrossRef] [PubMed]

20. Kunze, A.; Svedhem, S.; Kasemo, B. Lipid Transfer between Charged Supported Lipid Bilayers and Oppositely Charged Vesicles. Langmuir 2009, 25, 5146-5158. [CrossRef]

21. Tabaei, S.R.; Gillissen, J.J.J.; Vafaei, S.; Groves, J.T.; Cho, N.-J. Size-dependent, stochastic nature of lipid exchange between nano-vesicles and model membranes. Nanoscale 2016, 8, 13513-13520. [CrossRef]

22. Ohlsson, G.; Tigerström, A.; Höök, F.; Kasemo, B. Phase transitions in adsorbed lipid vesicles measured using a quartz crystal microbalance with dissipation monitoring. Soft Matter 2011, 7, 10749-10755. [CrossRef]

23. Neupane, S.; De Smet, Y.; Renner, F.U.; Losada-Pérez, P. Quartz Crystal Microbalance With Dissipation Monitoring: A Versatile Tool to Monitor Phase Transitions in Biomimetic Membranes. Front. Mater. 2018, 5, 46. [CrossRef]

24. Peschel, A.; Langhoff, A.; Uhl, E.; Dathathreyan, A.; Haindl, S.; Johannsmann, D.; Reviakine, I. Lipid phase behavior studied with a quartz crystal microbalance: A technique for biophysical studies with applications in screening. J. Chem. Phys. 2016, 145, 204904. [CrossRef]

25. Pramanik, S.K.; Seneca, S.; Ethirajan, A.; Neupane, S.; Renner, F.U.; Losada-Pérez, P. Ionic strength dependent vesicle adsorption and phase behavior of anionic phospholipids on a gold substrate. Biointerphases 2016, 11, 019006. [CrossRef] [PubMed]

26. Hasan, I.Y.; Mechler, A. Cholesterol Rich Domains Identified in Unilamellar Supported Biomimetic Membranes via Nano-Viscosity Measurements. Anal. Chem. 2016, 88, 5037-5041. [CrossRef] [PubMed]

27. Colombo, M.; Raposo, G.; Théry, C. Biogenesis, secretion, and intercellular interactions of exosomes and other extracellular vesicles. Annu. Rev. Cell Dev. Biol. 2014, 30, 255-289. [CrossRef] 
28. Enoki, T.A.; Henriques, V.B.; Lamy, M.T. Light scattering on the structural characterization of DMPG vesicles along the bilayer anomalous phase transition. Chem. Phys. Lipids 2012, 165, 826-837. [CrossRef]

29. Lipowsky, R.; Seifert, U. Adhesion of Vesicles and Membranes. Mol. Cryst. Liq. Cryst. 1991, 202, 17-25. [CrossRef]

30. Florin, E.-L.; Rief, M.; Lehmann, H.; Ludwig, M.; Dornmair, C.; Moy, V.; Gaub, H. Sensing specific molecular interactions with the atomic force microscope. Biosens. Bioelectron. 1995, 10, 895-901. [CrossRef]

31. Bibissidis, N.; Betlem, K.; Cordoyiannis, G.; Bonhorst, F.P.-V.; Goole, J.; Raval, J.; Daniel, M.; Góźdź, W.; Iglič, A.; Losada-Pérez, P. Correlation between adhesion strength and phase behaviour in solid-supported lipid membranes. J. Mol. Liq. 2020, $320,114492$. [CrossRef]

32. Lind, T.K.; Cárdenas, M. Understanding the formation of supported lipid bilayers via vesicle fusion-A case that exemplifies the need for the complementary method approach (Review). Biointerphases 2016, 11, 020801. [CrossRef]

33. Lee, C.-H.; Lin, W.-C.; Wang, J. All-optical measurements of the bending rigidity of lipid-vesicle membranes across structural phase transitions. Phys. Rev. E 2001, 64, 020901. [CrossRef]

34. Losada-Pérez, P.; Mertens, N.; De Medio-Vasconcelos, B.; Slenders, E.; Leys, J.; Peeters, M.; Van Grinsven, B.; Gruber, J.; Glorieux, C.; Pfeiffer, H.; et al. Phase Transitions of Binary Lipid Mixtures: A Combined Study by Adiabatic Scanning Calorimetry and Quartz Crystal Microbalance with Dissipation Monitoring. Adv. Condens. Matter Phys. 2015, 2015, 1-14. [CrossRef]

35. Leys, J.; Losada-Pérez, P.; Slenders, E.; Glorieux, C.; Thoen, J. Investigation of the melting behavior of the reference materials biphenyl and phenyl salicylate by a new type adiabatic scanning calorimeter. Thermochim. Acta 2014, 582, 68-76. [CrossRef]

36. Ebel, H.; Grabitz, P.; Heimburg, T. Enthalpy and Volume Changes in Lipid Membranes. I. The Proportionality of Heat and Volume Changes in the Lipid Melting Transition and Its Implication for the Elastic Constants. J. Phys. Chem. B 2001, 105, 7353-7360. [CrossRef]

37. Betlem, K.; Cordoyiannis, G.; Losada-Pérez, P. Supported Lipid Membranes at the Au-Buffer Interface by Solvent Exchange: The Effect of Initial Solvent Concentration. Phys. Status Solidi (a) 2020, 217, 1900837. [CrossRef]

38. Hasan, I.Y.; Mechler, A. Nanoviscosity Measurements Revealing Domain Formation in Biomimetic Membranes. Anal. Chem. 2017, 89, 1855-1862. [CrossRef]

39. Thilo, L. Kinetics of phospholipid exchange between bilayer membranes. Biochim. Biophys. Acta (BBA) - Biomembr. 1977, 469, 326-334. [CrossRef]

40. Bayerl, T.M.; Schmidt, C.F.; Sackmann, E. Kinetics of symmetric and asymmetric phospholipid transfer between small sonicated vesicles studied by high-sensitivity differential scanning calorimetry, NMR, electron microscopy, and dynamic light scattering. Biochemistry 1988, 27, 6078-6085. [CrossRef]

41. Rogers, J.R.; Espinoza-García, G.; Geissler, P.L. Membrane Hydrophobicity Determines the Activation Free Energy of Passive Lipid Transport. BioRxiv 2021. Available online: https:/ /www.biorxiv.org/content/10.1101/2021.03.17.435885v1 (accessed on 29 March 2021). [CrossRef]

42. Nakano, M.; Fukuda, M.; Kudo, T.; Endo, H.; Handa, T. Determination of Interbilayer and Transbilayer Lipid Transfers by Time-Resolved Small-Angle Neutron Scattering. Phys. Rev. Lett. 2007, 98, 238101. [CrossRef]

43. Neupane, S.; Betlem, K.; Renner, F.U.; Losada-Pérez, P. Solvent-Assisted Lipid Bilayer Formation on Au Surfaces: Effect of Lipid Concentration on Solid-Supported Membrane Formation. Phys. Status solidi (a) 2020. [CrossRef]

44. Li, M.; Chen, M.; Sheepwash, E.; Brosseau, C.L.; Li, H.; Pettinger, B.; Gruler, H.; Lipkowski, J. AFM Studies of Solid-Supported Lipid Bilayers Formed at a $\mathrm{Au}(111)$ Electrode Surface Using Vesicle Fusion and a Combination of Langmuir-Blodgett and Langmuir-Schaefer Techniques. Langmuir 2008, 24, 10313-10323. [CrossRef] [PubMed]

45. Dufrêne, Y.F.; Barger, W.R.; Green, A.J.-B.D.; Lee, G.U. Nanometer-Scale Surface Properties of Mixed Phospholipid Monolayers and Bilayers. Langmuir 1997, 13, 4779-4784. [CrossRef]

46. Redondo-Morata, L.; Giannotti, M.I.; Sanz, F. Influence of Cholesterol on the Phase Transition of Lipid Bilayers: A TemperatureControlled Force Spectroscopy Study. Langmuir 2012, 28, 12851-12860. [CrossRef] [PubMed] 